\title{
Inferring network structures via signal Lasso
}

\author{
Lei Shi $\odot,{ }^{1,2, *}, \dagger$ Chen Shen,${ }^{1, \dagger}$ Libin Jin, ${ }^{2, \dagger}$ Qi Shi, ${ }^{3}$ Zhen Wang, ${ }^{3, \dagger}$ and Stefano Boccaletti ${ }^{4,5,6}$ \\ ${ }^{1}$ School of Statistics and Mathematics, Yunnan University of Finance and Economics, Kunming 650221, China \\ ${ }^{2}$ Interdisciplinary Research Institute of Data Science, Shanghai Lixin University of Accounting and Finance, Shanghai 201209, China \\ ${ }^{3}$ Center for Optical Imagery Analysis and Learning, Northwestern Polytechnical University, Xi'an 710072, China \\ ${ }^{4}$ Institute for Complex Systems, CNR, Via Madonna del Piano 10, 50019 Florence, Italy \\ ${ }^{5}$ Unmanned Systems Research Institute, Northwestern Polytechnical University, Xi'an 710072, China \\ ${ }^{6}$ Moscow Institute of Physics and Technology, Institutskiy pereulok, Dolgoprudny, Moscow Region 141701, Russia
}

(Received 21 June 2021; accepted 1 December 2021; published 22 December 2021)

\begin{abstract}
Inferring the connectivity structure of networked systems from data is an extremely important task in many areas of science. Most real-world networks exhibit sparsely connected topologies, with links between nodes that in some cases may even be associated with a binary state ( 0 or 1 , denoting, respectively, the absence or presence of a connection). Such un-weighted topologies are elusive to classical reconstruction methods such as Lasso or compressed sensing techniques. We here introduce an approach called signal Lasso, in which the estimation of the signal parameter is subjected to 0 or 1 values. The theoretical properties and algorithm of the proposed method are studied in detail. Applications of the method are illustrated for an evolutionary game and synchronization dynamics in several synthetic and empirical networks, for which we show that our strategy is reliable and robust and outperforms the classical approaches in terms of accuracy and mean square errors.
\end{abstract}

DOI: 10.1103/PhysRevResearch.3.043210

\section{INTRODUCTION}

Complex networks have a wide range of applications in various fields of science [1-7]. In many cases, the pattern of the node-to-node interactions (the so-called underlying network topology) is unknown and needs to be retrieved, and therefore, uncovering the connectivity wiring of a distributed system's elementary units based on observable quantities obtained from experiments is a very relevant issue. Network reconstruction is an inverse problem and has received great attention recently [8-15]. Most existing approaches rely on the observation of the graph's dynamics (for instance, that exhibited by networked oscillators) as a result of continuous differential equations. However, gathering continuous-in-time information on the dynamical evolution of the graph is not always possible in social, economic, and even biological systems. Evolutionary-game-based dynamics, for instance, characterizes many relevant situations and is intrinsically discrete in time. In such a case, the problem can be transformed into a statistical linear model, with sparse and high-dimensional properties. The compressed sensing (CS) and the Lasso methods have been proposed for the purpose of

\footnotetext{
*1shi@ynufe.edu.cn

${ }^{\dagger}$ These authors contributed equally to this work.

‡W-zhen@nwpu.edu.cn
}

Published by the American Physical Society under the terms of the Creative Commons Attribution 4.0 International license. Further distribution of this work must maintain attribution to the author(s) and the published article's title, journal citation, and DOI. network reconstruction $[16,17]$. These two methods provide a good performance in uncovering the network topology, even for cases in which only a limited time series is observed, and the Lasso method is, furthermore, quite robust against various sources of noise which may affect the data.

Reconstructing the connectivity pattern of unweighted networks implies, however, estimating connection parameters between nodes which have true values of either 0 or 1 . All existing methods (including CS and Lasso) have the ability of shrinking the estimates to zero when networks are sparse but fail in compressing the estimates to 1 , and this latter fact unavoidably reduces their estimation accuracies. In this paper we proposed a method, which we call signal Lasso, able to uncover a graph's topology with a small amount of data. The main idea behind this method is to add a control term of the $L 1$ norm in the penalty function of Lasso that is able to shrink the estimates to 1 and therefore overcome the above limitation. The result is an estimate of the signal parameters with values exactly equal to 0 or 1 for nonexistent and existent links between nodes, respectively. We will show that our method is robust (at least as robust as Lasso) against noise and missing nodes due to the fact that a least-squares error control term is included. Validation and assessment of the method's robustness are illustrated with reference to the spatial ultimatum game and Kuramoto model in the synchronization dynamic for three typical classes of synthetic networks (Erdös-Rényi (ER) random [4], Watts-Strogatz (WS) small-world [5], and Barabási-Albert (BA) scale-free networks [6]) as well as for some empirical networks. High prediction accuracies are obtained also for extremely small data sets compared to the accuracies with the CS and Lasso methods. Therefore, our results are of potential interest for many applications in social, 
economic, and biological systems, all cases in which recovering hidden networks from very limited samples of data is required.

The problem under study can be framed as the solution of the following linear equation [16]:

$$
Y=\Phi X,
$$

where the $n \times p$ matrix $\Phi$ and the $n \times 1$ vector $Y$ are accessible, whereas $X$ is a parameter vector which is related to the adjacency matrix $\left[a_{i j}\right]$ of the network (with elements $a_{i j}=1$ for all pairs $i, j$ of connected nodes and $a_{i j}=0$ otherwise). Therefore, $X$ is a signal parameter with values of 1 or 0 , and the purpose of network reconstruction is to estimate $X$ from $Y$ and $\Phi$. Normally, the dimension of $X$ is much higher than the dimension of $Y$, and the problem displays a sparsity property due to the fact that only a few nonzero elements are present in $X[7,18]$. Recently, the Lasso method was proposed to find a solution of Eq. (1) from data, which is obtained by solving a convex optimization problem in view of a variable selection $[19,20]$. However, the Lasso method is able to reduce the parameter estimates of unimportant predictors in $X$ to zero, but the estimators of nonzero elements fail to be conducted towards their real values, 1. This latter feature of the methods causes the estimators of parameters with a value of 1 to have a rather low accuracy, as we will show in the present paper. Other methods, such as smoothly clipped absolute deviation (SCAD) [21], adaptive Lasso [22], group Lasso [23], and elastic net [24] and CS [25], which intrinsically focus on zero elements, suffer the same restriction as Lasso and therefore fail to give accurate descriptions of the nonzero elements of $X$.

\section{SIGNAL LASSO}

We exclude an intercept in model (1) for consistence with the model we study in this paper. The least-squares method for estimating $X$ is found by minimizing $\sum_{i=1}^{n}\left(y_{i}-\phi_{i}^{\prime} X\right)^{2}$, while the Lasso method [19] is subject to the constraint that $\sum_{i=1}^{n}\left|X_{i}\right|<t$, where $t>0$ is a tuning parameter and $\phi_{i}$ denotes the $i$ th row of $\Phi$. The solution of Lasso can be found by minimizing the following penalty likelihood:

$$
\frac{1}{2} \sum_{i=1}^{n}\left(y_{i}-\phi_{i}^{\prime} X\right)^{2}+\lambda \sum_{j=1}^{p}\left|X_{j}\right|
$$

with regulation parameter $\lambda$. However, in our problem, $X_{j}$ must be either 0 or 1 ; therefore, a method called signal Lasso is proposed by minimizing the following function:

$$
\frac{1}{2} \sum_{i=1}^{n}\left(y_{i}-\phi_{i}^{\prime} X\right)^{2}+\lambda\left[(1-\alpha) \sum_{j=1}^{p}\left|X_{j}\right|+\alpha \sum_{j=1}^{p}\left|X_{j}-1\right|\right],
$$

where $\mathbf{1}_{p}$ is a $p \times 1$ vector with all elements equal to $1,\|X\|_{k}$ is the $k$ norm of vector $X$ defined by $\|X\|_{k}=\left(\sum_{j=1}^{p}\left|X_{j}\right|^{k}\right)^{1 / k}$, and $\lambda>0$ and $\alpha \in(0,1)$ are two tuning parameters. We add the term of $\sum_{j=1}^{p}\left|X_{j}-1\right|$ in the penalty term since some elements of $X$ should be 1 . This is a compromise between the terms shrinking to 0 and 1 , and we hope some elements of $X$ will close be to 0 and others will be close to 1 . Minimization of Eq. (3) can lead to the solution of the optimal estimation of $X$. In general, it is impossible to make $X_{j}$ close to 0 and 1 at the same time; a common way in computational mathematics is to give a probability such as $\alpha$ to the signal group and $1-\alpha$ to the nonsignal group; as shown in Eq. (3), the value of $\alpha$ can be determined from the data set at hand.

Equation (3) can be rewritten in a more general form by

$$
\min _{X}\left\{\frac{1}{2}\|Y-\Phi X\|_{2}^{2}+\lambda_{1}\|X\|_{1}+\lambda_{2}\left\|X-\mathbf{1}_{p}\right\|_{1}\right\},
$$

where $\mathbf{1}_{p}$ is the $p \times 1$ vector with all elements equal to $1, \lambda_{1}$ and $\lambda_{2}$ are non-negative regularization parameters, and $\|X\|_{k}$ is the $k$ norm of the vector $X$. Furthermore, the presence of a least-squares term $\|Y-\Phi X\|_{2}^{2}$ in Eq. (4) makes the solution robust against noise. Since Eq. (4) is a convex optimization problem, the solution is unique, and some algorithms can be developed to solve it $[18,26]$. Moreover, it has to be noted that Eq. (4) reduces to the classical Lasso method for $\lambda_{2}=0$.

Let us now focus on the case in which the columns of $\Phi$ are orthogonal, with $\Phi^{\prime} \Phi=I_{p}\left(I_{p}\right.$ denotes the identity matrix of order $p$ ), and let us have $\delta_{1}=\lambda_{1}+\lambda_{2}$ and $\delta_{2}=\lambda_{1}-\lambda_{2}$. Then, for $k=1, \ldots, p$, the solution has the following form (see the Appendix A for full details):

$$
\hat{X}_{k}= \begin{cases}\left(\hat{X}_{k 0}+\delta_{1}\right)_{-}, & \hat{X}_{k 0} \leqslant 0 \\ \left(\hat{X}_{k 0}-\delta_{2}\right)_{+}, & 0<\hat{X}_{k 0} \leqslant 1+\delta_{2}, \\ \max \left\{1, \hat{X}_{k 0}-\delta_{1}\right\}, & \hat{X}_{j 0}>1+\delta_{2}\end{cases}
$$

where $\hat{X}_{k 0}$ and $\hat{X}_{k}$ are the $k$ th elements of $\hat{X}_{0}$ and $\hat{X}$, respectively, and $(B)_{+}\left[(B)_{-}\right]$in Eq. (5) denotes the positive (negative) part of the vector $B$, meaning that $(B)_{+}=B$ if $B \geqslant 0\left[(B)_{-}=B\right.$ if $\left.B \leqslant 0\right]$ and $(B)_{+}=0$ if $B<0\left[(B)_{-}=0\right.$ if $B>0]$. Note, furthermore, that there is a one-by-one transformation between $\left(\lambda_{1}, \lambda_{2}\right)$ and $\left(\delta_{1}, \delta_{2}\right)$. All details on the theoretical basis and algorithm of our method are given in Appendices A and B.

In Fig. 1 we report the solution $\hat{X}$ as a function of $\hat{X}_{0}$ for the Lasso method [Fig. 1(a)] and for the signal Lasso method [Figs. 1(b) and 1(c)]. From Fig. 1(a) one immediately sees that the Lasso method is able to shrink the parameter estimator $\hat{X}$ to zero for $\left|\hat{X}_{0}\right| \leqslant 0.6$ : the solution in this case is given by $\hat{X}_{k}=\operatorname{sgn}\left(\hat{X}_{k 0}\right)\left(\left|\hat{X}_{k 0}\right|-\lambda\right)_{+}$, where $\lambda=0.6$ and $\operatorname{sgn}(z)$ is a threshold function with $\operatorname{sgn}(z)=\operatorname{sign}(z)$ if $z \neq 0$ and is some value lying in $[-1,1]$. However, for $\left|\hat{X}_{k 0}\right|>0.6$, the classical Lasso method is able to translate the values only by a constant factor. Figure 1(b) reports the results of the signal Lasso method for $\lambda_{1}=0.6$ and $\lambda_{2}=0.4$ (this choice is equivalent to $\delta_{1}=1.0, \delta_{2}=0.2$ ). Some geometry properties of the signal Lasso method are apparent: values in the interval $-\delta_{1} \leqslant \hat{X}_{k 0} \leqslant \delta_{2}$ shrink to $\hat{X}=0$, and values satisfying $1+\delta_{2} \leqslant \hat{X}_{k 0} \leqslant 1+\delta_{1}$ shrink to 1 . Other values are translated by a constant. Therefore, this method is also able to compress larger values of the parameter estimator to 1 , resulting in an improvement of the estimation accuracy. Figure 1(c) reports the results for the case $\delta_{1}=1.8, \delta_{2}=0.4$, in which the lengths of intervals that shrink to 0 and 1 increase. Finally, in Fig. 1(d) we report the penalty function $\mathrm{PF}=\lambda_{1}\|X\|_{1}+\lambda_{2}\left\|X-\mathbf{1}_{p}\right\|_{1}$ vs $X$ for the same conditions as in Fig. 1(b) in a way that the shrinking directions toward 0 and 1 are visible. The constraint region in the signal Lasso method is a polygon with four to eight corners (see Fig. 6 in Appendix A for full details). Figure 2 shows the estimation 

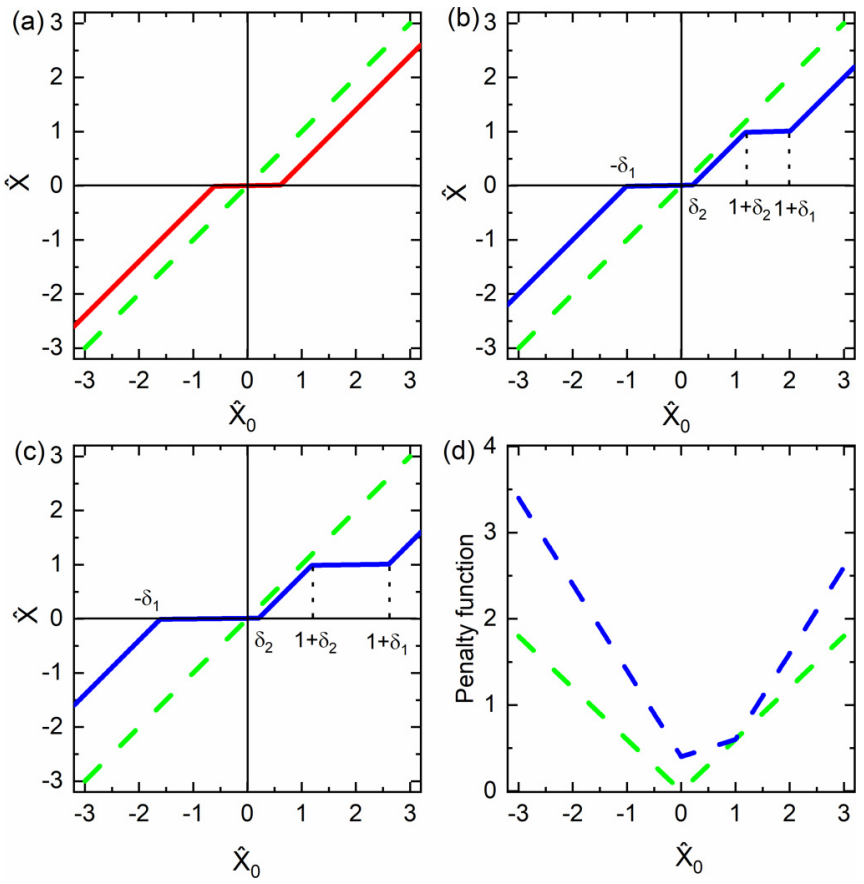

FIG. 1. (a) Solution $\hat{X}$ (red curve) for the Lasso method $(\lambda=$ $0.6)$ vs the solution $\hat{X}_{0}$ obtained under least squares, (b) solution $\hat{X}$ (blue curve) for the signal Lasso method $\left(\delta_{1}=1, \delta_{2}=0.2\right)$ vs $\hat{X}_{0}$, and (c) same as (b), but for $\delta_{1}=2$ and $\delta_{2}=0.4$. In all three panels, we draw a green line, $\hat{X}=\hat{X}_{0}$, for reference. (d) Penalty functions (see text for definition) of the signal Lasso method (blue line) for $\delta_{1}=1, \delta_{2}=0.2$ and of the Lasso method (green line) for $\lambda=0.6$.

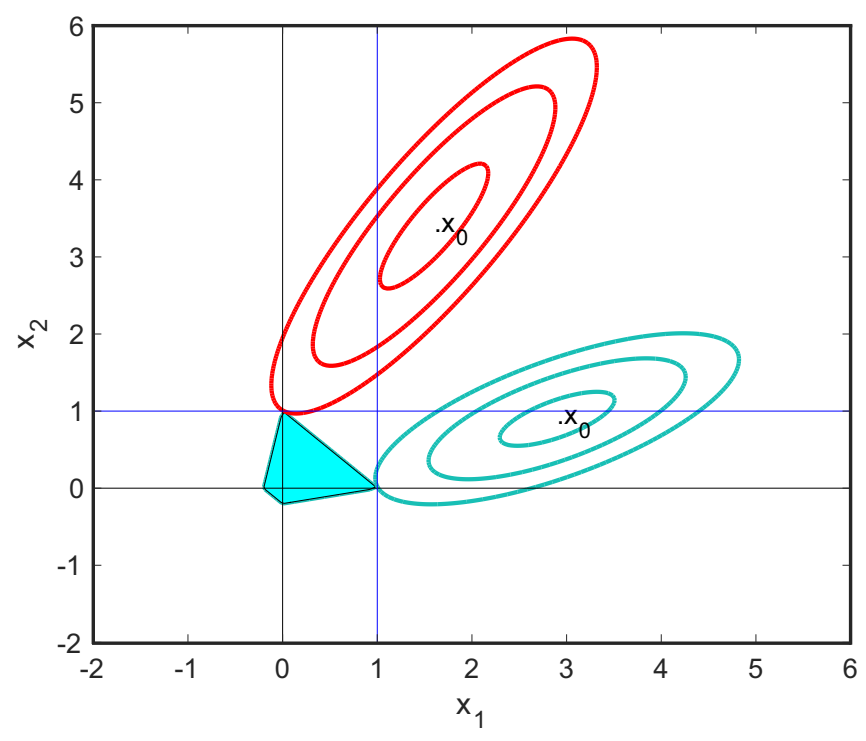

FIG. 2. Sketch of how estimation is obtained in the signal Lasso method. The solid blue area is the constraint region $\lambda_{1} \sum_{j=1}^{2}\left|x_{j}\right|+$ $\lambda_{2} \sum_{j=1}^{2}\left|x_{j}-1\right| \leqslant 1$, with $\lambda_{1}=0.6, \lambda_{2}=0.4$, while the ellipses are the contours of the residual sum of squares in two situations, centered around the solutions $X_{0}$ under least squares. In both cases, the method finds the first point where the elliptical contours hit the corner of the constraint region, which corresponds to $(0,1)$ for the upper ellipses and $(1,0)$ for the lower ellipses. Interested readers can compare this scenario with the case of the Lasso method (reported in Ref. [20]). picture, where the solution [such as $(0,1)$ or $(1,0)$ in a twodimensional case] is found from the elliptical residual sum of the square hitting the corner of the constraint region. When the columns of $\Phi$ are not orthogonal and $p$ is sufficiently larger than $n$, the solution can be found by convex optimization algorithms. The choice of $\lambda_{1}$ and $\lambda_{2}$ (or, equivalently, $\delta_{1}$ and $\delta_{2}$ ) is guided by cross-validation methods (see Appendix C for full details ).

\section{RECONSTRUCTION OF CONNECTIVITY BASED ON AN EVOLUTIONARY GAME}

In what follows, we apply the method to reconstruct the connectivity pattern of players of the spatial ultimatum game (SUG). In such a framework, each node of a network of size $N$ represents a player, and links of the graph stand for game relationships between players. In the SUG [28,29], each player $i$ is assigned an arbitrary strategy pair $\left(p_{i}, q_{i}\right)$, with $p_{i}, q_{i} \in$ $[0,1]$, which are drawn from a uniform distribution and which represent their offering and acceptance level, respectively. The payoff $P_{i j}$ that player $i$ obtains from playing with player $j$ is calculated as follows:

$$
P_{i j}=\left\{\begin{array}{lll}
p_{j}+1-p_{i}, & p_{i} \geqslant q_{j}, & p_{j} \geqslant q_{i}, \\
1-p_{i}, & p_{i} \geqslant q_{j}, & p_{j}<q_{i}, \\
p_{j}, & p_{i}<q_{j}, \quad p_{j} \geqslant q_{i}, \\
0, & p_{i}<q_{j}, \quad p_{j}<q_{i} .
\end{array}\right.
$$

Then, the total payoff $F_{i}$ is calculated as $F_{i}=\sum_{j} a_{i j} P_{i j}$, where $a_{i j}=1$ if node $\mathrm{i}$ and node $\mathrm{j}$ is connected and zero otherwise. In each round of the game, each player first calculates their total payoff and then imitates with a certain probability the two strategies $(p, q)$ of a randomly selected player in their direct neighborhood. Precisely, player $x$ adopts the strategy of player $y$ with probability $W=1 /\left\{1+\exp \left[\left(F_{x}-F_{y}\right) / K\right]\right\}[30,31]$. In order to make the model more realistic, we also account for mutation: in each round of the game, all players adjust their strategy $(p, q)$ according to $\left(p_{i}(t+1), q_{i}(t+1)\right)=\left(p_{i}(t)+\right.$ $\left.\delta, q_{i}(t)+\delta\right)$, where $\delta \in[-\epsilon, \epsilon]$ is a small random number (in our examples $\epsilon=0.05)$.

Now $F_{i}=\sum_{j=1, j \neq i}^{N} a_{i j} P_{i j}$ can be written as a linear regression model,

$$
Y_{i}=\Phi_{i} X_{i}
$$

where $Y_{i}=\left(F_{i}\left(t_{1}\right), F_{i}\left(t_{2}\right), \ldots, F_{i}\left(t_{L}\right)\right)^{\prime}, \quad X_{i}=\left(a_{i 1}, \ldots, a_{i N}\right)^{\prime}$, and $\Phi_{i}$ has the form

$$
\left(\begin{array}{cccccc}
P_{i 1}\left(t_{1}\right) & \ldots & P_{i, i-1}\left(t_{1}\right) & P_{i, i+1}\left(t_{1}\right) & \ldots & P_{i_{N}}\left(t_{1}\right) \\
P_{i 1}\left(t_{2}\right) & \ldots & P_{i, i-1}\left(t_{2}\right) & P_{i, i+1}\left(t_{2}\right) & \ldots & P_{i_{N}}\left(t_{2}\right) \\
\vdots & \vdots & \vdots & \vdots & \vdots & \vdots \\
P_{i 1}\left(t_{L}\right) & \ldots & P_{i, i-1}\left(t_{L}\right) & P_{i, i+1}\left(t_{L}\right) & \ldots & P_{i_{N}}\left(t_{L}\right)
\end{array}\right) .
$$

Let $Y=\Phi X$, where $Y=\left(Y_{1}^{\prime}, \ldots, Y_{N}^{\prime}\right)^{\prime}, X=\left(X_{1}^{\prime}, \ldots, X_{N}^{\prime}\right)^{\prime}$, $\Phi=\operatorname{diag}\left(\Phi_{1}, \Phi_{2}, \ldots, \Phi_{N}\right)$; then Eq. (7) can be converted to the general form of Eq. (1). We suppose here that all total payoffs are accessible at $L$ successive times, so that one can write a linear regression model of the form of Eq. (1).

In order to quantify the performance of the method we use the mean square error (MSE) to measure how close the inferred adjacency matrix $A^{\star}$ is to the true matrix $A$. Specifically, 

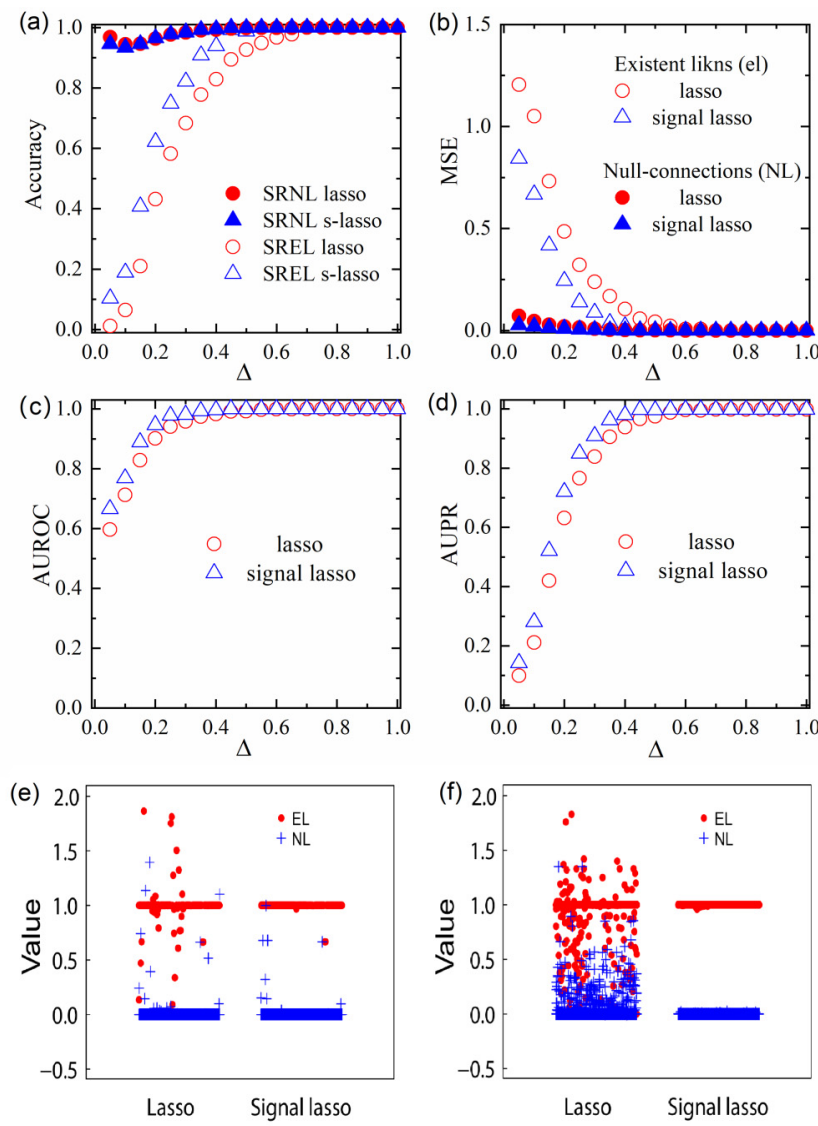

FIG. 3. Accuracy of the reconstruction vs $\Delta=L / N$, attained by the Lasso and signal Lasso methods. (a)-(d) refer to a SUG played on top of Barabási-Albert (BA) scale-free networks and report (a) the SREL and SRNL criteria, (b) the MSE criterion, (c) the AUROC criterion, and (d) the AUPR criterion (see text for all definitions). Reconstructed values of the elements of the vector $X$ obtained by the Lasso and signal Lasso methods for a SUG played (e) on top of the karate club network with $\Delta=0.5$ and (f) on Erdös-Rényi random networks with an average degree of 12 for $\Delta=0.4$. $N$ is equal to 100 and 34 for the BA and karate club networks, respectively.

we use the following equation for existent and nonexistent links:

$$
\operatorname{MSE}_{k}=\frac{\sum_{i}^{N} \sum_{j \in \Omega_{k}}\left(a_{i j}-a_{i j}^{\star}\right)^{2}}{N_{k}}, \quad k=1,2,
$$

where $\Omega_{1}\left(\Omega_{2}\right)$ is the set of existent (nonexistent) links and $a_{i j}^{\star}$ $\left(a_{i j}\right)$ are the elements of $A^{\star}(A)$. For a complete comparison, we compute the area under the receiver operating characteristic curve (AUROC) and the area under the precision recall curve (AUPR), as defined in Ref. [16], as well as the success rates for the detection of existing links (SREL) and nonexisting links (SRNL), as defined in Ref. [17].

Figure 3 reports a comparison between the accuracy attained by the signal Lasso and Lasso methods for BA scale-free networks with $N=100$ for the criteria of SREL and SRNL [Fig. 3(a)], MSE [Fig. 3(b)], AUROC [Fig. 3(c)], and AUPR [Fig. 3(d)] adopted. The amount of data is scaled as $\Delta=L / N$. One clearly sees that the signal Lasso method always improves the reconstruction accuracy (compared with the Lasso method), no matter which comparison criterion is adopted. For instance (and as an example), at $\Delta=0.4$ we have $\mathrm{MSE}=0.02$ for signal Lasso and $\mathrm{MSE}=0.1$ for Lasso. We also used the karate club network [32] and ER networks with average degree $\langle k\rangle=12$. The results are shown in Figs. 3(e) and 3(f), respectively. As predicted, the signal Lasso method effectively shrinks the parameter values of $X$ to 0 or 1 in both cases, while the performance of the Lasso method is poorer. A more complete comparison is reported in Table I, where we compare our method with Lasso and CS in terms of the minimal number of data (the maximum $\Delta$ ) needed to achieve an accuracy value of at least 0.05 in both $\mathrm{MSE}_{1}$ (associated with the reconstruction task of existent links) and $\mathrm{MSE}_{2}$ (associated with the reconstruction task of nonexistent links) for different types of networks. The performance of signal Lasso is always better than the other two methods, and the advantage is more prominent for networks with a higher average degree. When a Gaussian noise is present, the advantage is not very big, yet the highest reconstruction accuracy is obtained for the signal Lasso method. In Table II the same comparison is done for some real-world networks, with similar and consistent results. For more details on these latter results, we refer the interested reader to the Supplemental Material.

\section{RECONSTRUCTION OF CONNECTIVITY BASED ON THE SYNCHRONIZATION MODEL}

Next, we study the reconstruction of the network in the synchronization model [35-37]. We employ the simple Kuramoto model for the illustration, which has the following governing equation:

$$
\frac{d \theta_{i}}{d t}=\omega_{i}+c \sum_{j=1}^{N} a_{i j} \sin \left(\theta_{j}-\theta_{i}\right),
$$

with $i=1, \ldots, N$, where the system is composed of $N$ oscillators with phase $\theta_{i}$ and coupling strength $c$, each of the oscillators has its own intrinsic natural frequency $\omega_{i}$, and $a_{i j}$ is the adjacency matrix of a given network and needs to be estimated in the network reconstruction.

To solve the above equation, the Euler method was employed to generate time series with an equal time step $h$. The time increment of $\theta$ is used to approximate $\dot{\theta}=d \theta_{i} / d t$; in other words $\dot{\theta}=[\theta(t+h)-\theta(t)] / h$. Then we have the following update formula:

$$
\frac{\left[\theta_{i}(t+h)-\theta_{i}(t)\right]}{h}=\omega_{i}+c \sum_{j=1}^{N} a_{i j} * \sin \left[\theta_{j}(t)-\theta_{i}(t)\right] .
$$

Once initial values of $\theta_{i}(0)$ for $i=1, \ldots, N$ are given, the estimator of $\theta_{i}$ for $i=1, \ldots, N$ can be obtained iteratively, and a sequence of time series data is presented. Let $Y_{i}=$ $\left(y_{i 1}, \ldots, y_{i L}\right)^{\prime}, y_{i t}=\left[\theta_{i}(t+h)-\theta_{i}(t)\right] / h$,

$$
\Phi_{i}=\left(\begin{array}{cccc}
\phi_{i 1}(1) & \phi_{i, 2}(1) & \cdots & \phi_{i_{N}}(1) \\
\phi_{i 1}(2) & \phi_{i, 2}(2) & \cdots & \phi_{i_{N}}(2) \\
\vdots & \vdots & \vdots & \vdots \\
\phi_{i 1}(L) & \phi_{i, 2}(L) & \cdots & \phi_{i_{N}}(L)
\end{array}\right),
$$


TABLE I. Maximum value of the data ratio $\Delta$ which simultaneously ensures a value of at least 0.95 for the accuracy in reconstructing existent and nonexistent links. The methods compared are the compressed sensing (CS), Lasso, and signal Lasso methods. The comparison is made with a SUG played on scale-free networks (BA), small-world Watts-Strogatz networks, and random ER graphs. All results refer to averages over 10 independent realizations. $N$ is the network size, $\langle k\rangle$ is the average degree of the network, and $\sigma$ is the amplitude of the Gaussian noise.

\begin{tabular}{|c|c|c|c|c|c|}
\hline$N$ & $\langle k\rangle$ & $\sigma$ & $\begin{array}{c}\text { SF } \\
\text { CS/Lasso/signal Lasso }\end{array}$ & $\begin{array}{c}\text { WS } \\
\text { CS/Lasso/signal Lasso }\end{array}$ & $\begin{array}{c}\text { ER } \\
\text { CS/Lasso/signal Lasso }\end{array}$ \\
\hline 100 & 6 & 0 & $0.44 / 0.44 / 0.34$ & $0.26 / 0.26 / 0.24$ & $0.29 / 0.29 / 0.27$ \\
\hline 100 & 6 & 0.05 & $\operatorname{nan} / 0.49 / 0.4$ & $\operatorname{nan} / 0.36 / 0.33$ & $\operatorname{nan} / 0.34 / 0.34$ \\
\hline 100 & 6 & 0.3 & $\operatorname{nan} / 1.5 / 1.4$ & $\operatorname{nan} / 1.2 / 1.2$ & $\operatorname{nan} / 1.4 / 1.4$ \\
\hline 100 & 12 & 0 & $0.55 / 0.55 / 0.4$ & $0.39 / 0.39 / 0.36$ & $0.39 / 0.39 / 0.36$ \\
\hline 100 & 18 & 0 & $0.60 / 0.60 / 0.42$ & $0.43 / 0.43 / 0.39$ & $0.46 / 0.46 / 0.41$ \\
\hline 100 & 30 & 0 & $0.68 / 0.68 / 0.46$ & $0.55 / 0.55 / 0.49$ & $0.59 / 0.59 / 0.5$ \\
\hline
\end{tabular}

where $\phi_{i j}(t)=c \sin \left[\theta_{j}(t)-\theta_{i}(t)\right], X_{i}=\left(a_{i 1}, \ldots, a_{i N}\right)^{\prime}$; then,

$$
Y_{i}=\omega_{i} \mathbf{1}_{L}+\Phi_{i} X_{i}
$$

where $\mathbf{1}_{L}$ denotes an $L \times 1$ vector with all elements being 1 . Equation (10) can be stacked as a linear model as $Y=Z \omega+$ $\Phi X$, where $Y=\left(Y_{1}^{\prime}, \ldots, Y_{N}^{\prime}\right)^{\prime}, \Phi=\operatorname{diag}\left(\Phi_{1}, \Phi_{2}, \ldots, \Phi_{N}\right)$, $Z=I_{N} \otimes \mathbf{1}_{L}$, where $\otimes$ denotes the Kronecker product and $I_{N}$ is an identity matrix with order $N$. The method proposed here can be modified to give a solution slightly different from Eq. (4) since there is a heterogeneity parameter $\omega_{i}$. The solution of $X$ and $\omega$ using the signal Lasso method can be obtained by minimizing

$$
\min _{X, \omega}\left\{\frac{1}{2}\|Y-Z \omega-\Phi X\|_{2}^{2}+\lambda_{1}\|X\|_{1}+\lambda_{2}\left\|X-\mathbf{1}_{p}\right\|_{1}\right\} .
$$

Note that parameter $\omega$ does not shrink in minimization and needs to be estimated as an intrinsic natural frequency. The algorithm can be modified slightly from that in Sec. II.

We generate time series using the Euler method from Eq. (9) with equal time steps of 0.01 , where $\omega_{i}$ is generated from a uniform random variable in the range $[-0.5,0.5]$. Figure 4 gives the results of the reconstruction accuracy (SREL, SRNL, and MSE) in the ER, WS, and BA networks for $N=30,60$ and coupling strength $c=10$ (see Ref. [37] for a setting similar to this model), and the results clearly shown the signal Lasso method outperforms Lasso methods.

The scenarios with noise in this model can be similarly conducted; for example, we can generate the data from a normal distribution with mean zero and variance $\sigma^{2}$ and calculate the reconstruction accuracy (SREL, SRNL, and MSE) in terms of the ER, WS, and BA networks. We find that the reconstructions are more difficult with noise than those

TABLE II. Same as Table I, but here SUG was played on some real networks [32-34]. All results are averages over 10 independent realizations. $N$ is the network size, and $\langle k\rangle$ is the average degree of the given network.

\begin{tabular}{lrrccc}
\hline \hline Network & \multicolumn{1}{c}{$N$} & $\langle k\rangle$ & CS & Lasso & Signal Lasso \\
\hline Karate & 34 & 4.6 & 0.62 & 0.62 & 0.45 \\
Dolphins & 62 & 5.1 & 0.38 & 0.38 & 0.34 \\
Football & 115 & 10.7 & 0.29 & 0.29 & 0.27 \\
\hline \hline
\end{tabular}

without noise; however, they all show that the signal Lasso method is superior to the Lasso method.

\section{RECONSTRUCTION OF CONNECTIVITY BASED ON A HUMAN BEHAVIOR EXPERIMENT}

In this section, we present the results of social network reconstruction using real data from a human behavior experiment [38], whose purpose is to study the impact of punishment on network reciprocity. A total of 135 participants from the Yunnan University of Finance and Economics and the Tianjin University of Finance Economics took part in the experiments, and three trials were separately designed and carried out. In treatment I, 35 participants from the Tianjin University of Finance and Economics played an iterative prisoner's dilemma game with punishment on the static ring network with four neighbors, in which there are 35 nodes and 140 links. For the other two treatments, 100 participants from the Yunnan University of Finance and Economics were invited to participate in an iterated prisoner's dilemma experiment. Treatment II was implemented on a homogeneous random network with a degree of 4 , which has 50 nodes and 200 links. In treatment III, each player was placed on the heterogeneous random network, in which the degree of half the nodes is 3 and the degree of the other half of the nodes is 5 ; there are 50 nodes and 200 links. The network structures are illustrated in Fig. 5(a). The employed payoff matrices are given in Table III. In each round of the treatment, each player played with their direct neighbors to gain their payoff and updated their strategy to optimize their future payoff. The number of interactions in each session was set to 50, and the number of interactions was undisclosed until the session ended. In order to solve the network reconstruction problem in these examples, we thus recorded all the strategies and payoffs generated in the experiment, and this information is the available database for the reconstruction. Through this database, we can easily construct a linear regression model and obtain the reconstruction results under the Lasso and signal Lasso methods. The results are summarized in Figs. 5(b)-5(d). We observe that the signal Lasso method reaches higher accuracy than the Lasso method. The improvement from using signal Lasso is especially obvious for the data set with fewer data in the top row of Fig. 5 (with a relative length of about 0.5 ; treatment I) 

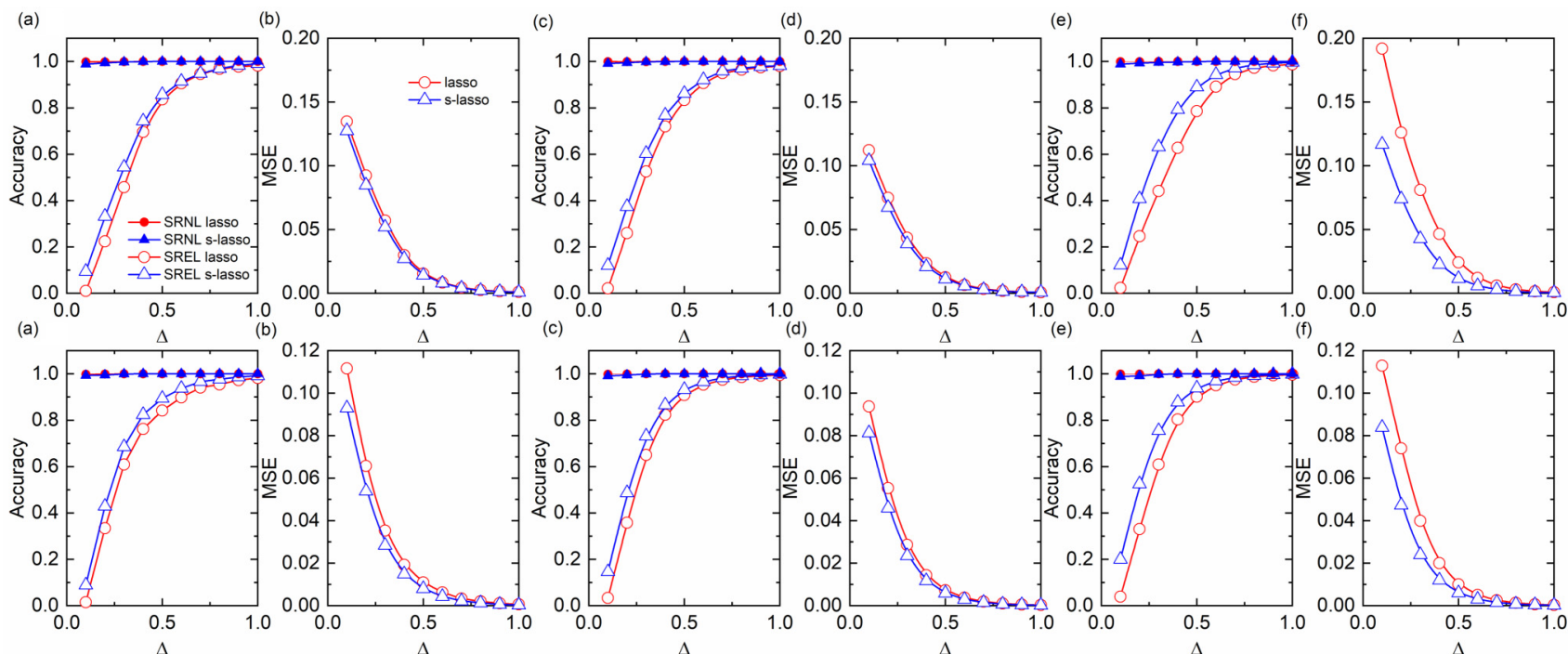

FIG. 4. Accuracy of the reconstruction vs $\Delta=L / N$ for the Kuramoto model attained by the Lasso and signal Lasso methods. (a) and (b) refer to Erdös-Rényi random networks with an average degree of 6. (c) and (d) refer to Barabási-Albert scale-free networks, and (e) and (f) refer to the small-world (WS) network. We report (a), (c), and (e) the SREL and SRNL criteria and (b), (d), and (f) the MSE criteria, where the top row is for network size $N=30, c=10$ and the bottom row is for network size $N=60, c=10$. Here we list only the total MSE and no longer distinguish the cases of existing links and nonexisting links. Each point is averaged over 50 simulations.
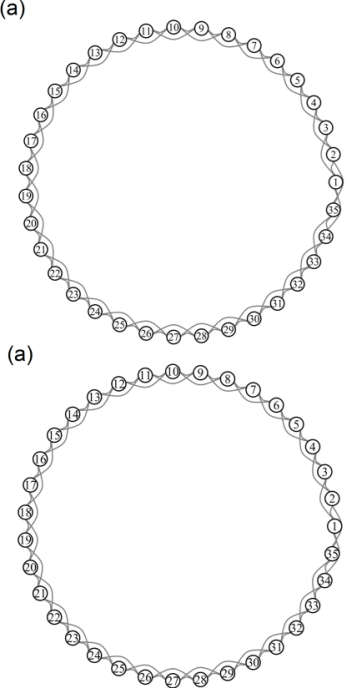

(a)

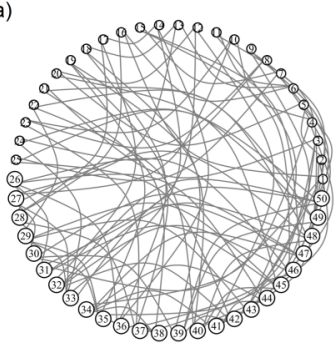

(b)

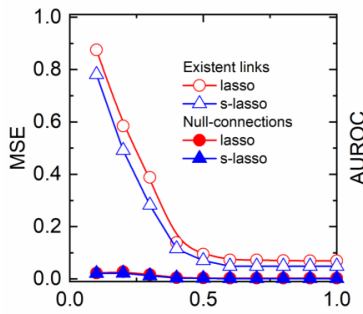

(b)

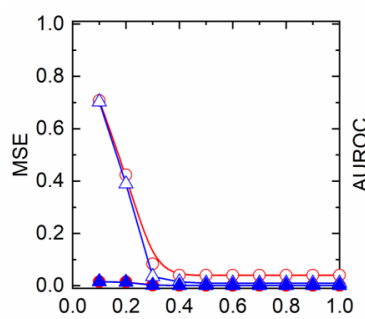

(b)

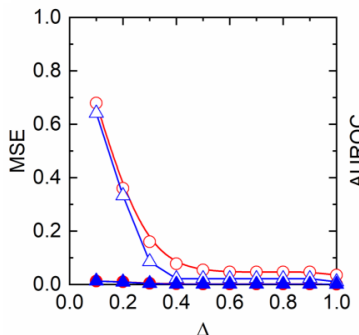

(c)

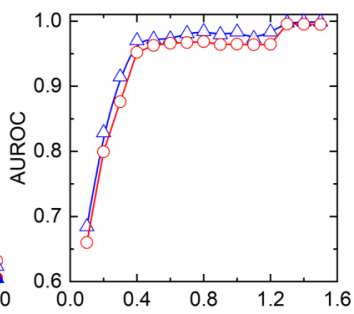

(c)

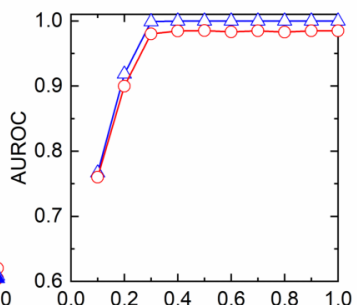

(c)

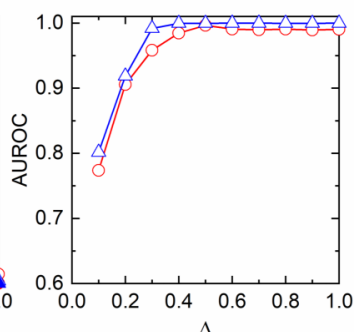

(d)

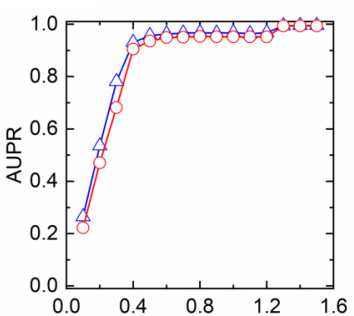

(d)

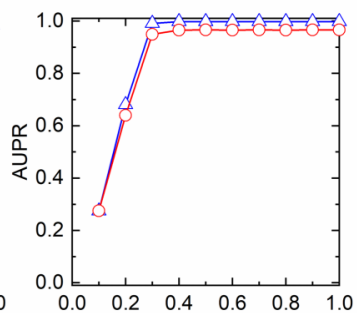

(d)

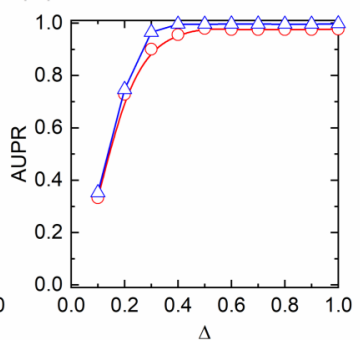

FIG. 5. Top: The results of the experimental ring network, where (a) is the scheme of the experiment and (b)-(d) refer to the MSE criterion, AUROC criterion, and AUPR criterion, respectively. There are 35 nodes and 140 links, and the degree of each node is 4. Middle: The results of the experimental homogeneous random network, where (a) is the scheme of the experiment and (b)-(d) refer to the MSE criterion, AUROC criterion, and AUPR criterion. There are 50 nodes and 200 links, and the degree of each node is 4. Bottom: The results of the experimental heterogeneous random network, where (a) is the scheme of the experiment and (b)-(d) refer to the MSE criterion, AUROC criterion, and AUPR criterion. There are 50 nodes and 200 links, and the average degree of each node is 4. 
TABLE III. The payoff matrices of the prisoner's dilemma game with a punishment option (treatment I) and the standard prisoner's dilemma (treatments II and III) are shown.

\begin{tabular}{lccc}
\hline \hline & \multicolumn{3}{c}{ Game with punishment option } \\
\cline { 2 - 4 } & $\mathrm{C}$ & $\mathrm{D}$ & $\mathrm{P}$ \\
\hline $\mathrm{C}$ & 2 & -2 & -5 \\
$\mathrm{D}$ & 4 & 0 & -3 \\
$\mathrm{P}$ & 2 & -2 & -5 \\
& & Standard game & \\
\cline { 2 - 4 } & $\mathrm{C}$ & $\mathrm{D}$ & \\
\hline $\mathrm{C}$ & 4 & -2 & \\
$\mathrm{D}$ & 6 & 0 & \\
\hline \hline
\end{tabular}

and the bottom row of Fig. 5 (with a relative length of about 0.4 ; treatment III).

\section{CONCLUSIONS}

To conclude, we introduced a method (signal Lasso) for the estimation problem of signal parameters in the area of network reconstruction. By adding a control term of an $L 1$ norm to shrink the parameters to 1 in the penalty function of Lasso, the estimated signal parameters can be compressed to 0 or 1 , which ensures higher reconstruction accuracies compared with the Lasso and compressed sensing methods. The detailed theoretical properties of the method (including the geometric interpretation and algorithms) are presented in Appendices A and B. For some complicated models with the common feature that the estimated parameters have signal properties, after some modifications such as replacing the least-squares term with more suitable measures (for instance, the likelihood function), the problem could be solved effectively using method suggested in this paper.

It is noteworthy that our method is not limited to the two models considered in this paper. For networked binary-state dynamical data [39], including the susceptibleinfected-susceptible (SIS) model, majority vote model, and language model, some problems can be converted to the models indexed with signal parameters [40-42]; thus, the method suggested in this paper can be used to obtain more efficient results. This method can also be used with data collected in real-world networks, for gene regulatory network reconstruction from expression data in systems biology $[43,44]$, to infer various functional networks in the human brain [45], and for uncovering organizational networks in the social and economic sciences. Due to its robustness against noise and general good performance, our method therefore may have wide applications. For large-scale networks, some dimensional reduction methods can be used first to overcome the dimension curse, and then the method suggested here can be employed to get the solutions [46].

It is well known that Lasso has some limitations, although it is a very innovative and effective technique for simultaneous continuous shrinkage and automatic variable selection. When there are group effects in the data set such that some variables in a group are highly correlated, Lasso tends to select only one variable from the group arbitrarily [24]. This limitation might exist in our signal Lasso because we do not consider this special phenomenon and focus only on detecting signal parameter. The elastic net is an improved version for solving this problem [24] and performs better than Lasso in some cases, but it is incapable of detecting the signal parameter, which is achieved by the signal Lasso method we proposed here. Some comparisons of signal Lasso with elastic net are given in Appendix D and the Supplemental Material [27] and confirm our conclusion. However, overcoming the identification of signal parameters in the case of high correlation of variables is an interesting issue and will be the topic of our research in the future.

\section{ACKNOWLEDGMENTS}

We thank Dr. M. Jusup and Dr. D. Yu for valuable discussions. The research was supported by the $\mathrm{Na}$ tional Natural Science Foundation of China (Grants No. 11931015, No. 11671348, No. 11801370, No. U1803263, No. 81961138010), the National Natural Science Foundation for Distinguished Young Scholars (Grant No. 62025602), and the Fok Ying-Tong Education Foundation of China (Grant No. 171105).

\section{APPENDIX A: PROOF OF THE RESULTS IN SIGNAL LASSO}

We rewrite the above likelihood function in a general matrix form as

$$
\begin{aligned}
& L\left(X, \lambda_{1}, \lambda_{2} \mid Y, \Phi\right) \\
& \quad=\frac{1}{2}\|Y-\Phi X\|_{2}^{2}+\lambda_{1}\|X\|_{1}+\lambda_{2}\left\|X-\mathbf{1}_{p}\right\|_{1},
\end{aligned}
$$

where $\lambda_{1}, \lambda_{2} \geqslant 0$ are two tuning parameters. $\operatorname{PF}(x)=$ $\lambda_{1}\|x\|_{1}+\lambda_{2}\left\|x-\mathbf{1}_{p}\right\|_{1}$ is called the penalty function. Figures 6(a)-6(d) list the constraint regions $\operatorname{PF}(x)=c$ of four different shrink estimation methods; the first three are wellknown methods: ridge regression [47], Lasso estimation, and elastic net estimation [24]. The elastic net method is a balance between ridge regression and the Lasso method. Our signal Lasso, as shown in Fig. 6(d), has edges less than or equal to 8 depending on the values of $c$.

In order to study the geometry of signal Lasso, we assume that the columns of $\Phi$ are orthogonal to each other and $p<n$. The ordinary least-squares estimate in this special case then has the form $\hat{X}_{0}=\Phi^{\top} Y$. Let $\hat{Y}_{0}=\Phi \hat{X}_{0}$; we then have

$$
\begin{aligned}
L\left(X, \lambda_{1}, \lambda_{2} \mid Y, \Phi\right)= & \frac{1}{2}\left\|Y-\hat{Y}_{0}\right\|_{2}^{2}+\frac{1}{2}\left\|X-\hat{X}_{0}\right\|_{2}^{2} \\
& +\lambda_{1}\|X\|_{1}+\lambda_{2}\left\|X-\mathbf{1}_{p}\right\|_{1} .
\end{aligned}
$$

Note that the first term on the right is constant with respect to $X$ and $\left\|\Phi\left(X-\hat{X}_{0}\right)\right\|_{2}^{2}=\left\|\left(X-\hat{X}_{0}\right)\right\|_{2}^{2}$. Using the fact [20] that

$$
\begin{aligned}
\frac{\partial|x|}{\partial x} & =\frac{\partial[\operatorname{sgn}(x) x]}{\partial x} \\
& = \begin{cases}1, & x>0, \\
-1, & x<0, \\
\text { some values in }[-1,1], & x=0,\end{cases}
\end{aligned}
$$



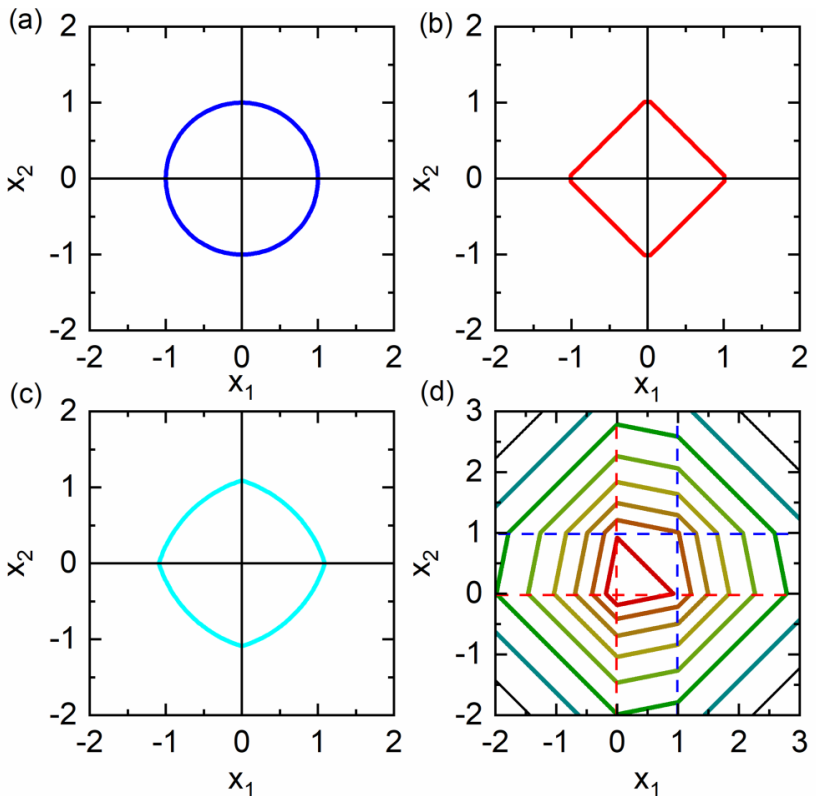

FIG. 6. Constraint regions of $\mathrm{PF}(x)=c$ for some constant $c$ under four Lasso-type estimation methods in the two-dimensional case $(p=2)$. (a) Ridge regression with penalty function $\operatorname{PF}(x)=$ $\sum_{j=1}^{2} x_{j}^{2}$. (b) Lasso estimation with penalty function $\operatorname{PF}(x)=$ $\sum_{j=1}^{2}\left|x_{j}\right|$. (c) Elastic net estimation with penalty function $\operatorname{PF}(x)=$ $\frac{1}{2} \alpha \sum_{j=1}^{2} x_{j}^{2}+(1-\alpha) \sum_{j=1}^{2}\left|x_{j}\right|, \alpha=0.5$. (d) Signal Lasso with penalty function $\operatorname{PF}(x)=\lambda_{1} \sum_{j=1}^{2}\left|x_{j}\right|+\lambda_{2} \sum_{j=1}^{2}\left|x_{j}-1\right|$ for different values of $c$ (to show the pattern for different $c$ ), where $\lambda_{1}=$ $0.6, \lambda_{2}=0.4$

and differentiating $L\left(X, \lambda_{1}, \lambda_{2} \mid Y, \Phi\right)$ with respect to $X$ and setting it to zero, after some calculations we have

$$
\hat{X}_{k}= \begin{cases}\left(\hat{X}_{k 0}+\delta_{1}\right)_{-}, & \hat{X}_{k 0} \leqslant 0, \\ \left(\hat{X}_{k 0}-\delta_{2}\right)_{+}, & 0<\hat{X}_{k 0} \leqslant 1+\delta_{2}, \\ \max \left\{1, \hat{X}_{k 0}-\delta_{1}\right\}, & \hat{X}_{k 0}>1+\delta_{2},\end{cases}
$$

for $k=1, \ldots, p$, where $\delta_{1}=\lambda_{1}+\lambda_{2}$ and $\delta_{2}=\lambda_{1}-\lambda_{2} ; \hat{X}_{k 0}$ and $\hat{X}_{k}$ are the $k$ th elements of $\hat{X}_{0}$ and $\hat{X}$, respectively; and $B_{+}$ denotes the positive part of $B$, meaning that $B_{+}=B$ if $B \geqslant 0$ and is zero otherwise. $B_{-}$is defined similarly to the negative part of $B$.

If an intercept needs to be included in the model, our method is also available. Since the intercept will not shrink, we simply center the covariates $\phi_{i j}$, and then the intercept can be replaced by $\bar{y}=\sum_{i=1}^{n} y_{i} / n$. Having solved for this optimal estimator, it remains to compute estimators of other coefficients using signal Lasso.

\section{APPENDIX B: ALGORITHM AND COMPUTATION}

It is noted that the penalty function $\operatorname{PF}(x)=$ $\lambda_{1} \sum_{j=1}^{p}\left|x_{j}\right|+\lambda_{2} \sum_{j=1}^{p}\left|x_{j}-1\right|$ is a convex function. Thus, the optimization problem in (4) does not suffer from the multiple local minimal issue, and its global minimizer can be efficiently solved. A variety of algorithms are available in some software packages with a convex optimization program and can be used to calculate the estimation of $X$ in the signal Lasso method. However, we can provide an alternative algorithm for signal Lasso using the coordinate descent method [20], like that in the Lasso estimation method. Below we give more detail.

The coordinate descent method is an iterative algorithm that updates the estimator by choosing a single coordinate to update and then performing a univariate minimization over this coordinate. Differentiating (4) with respect to $X_{k}$ and setting it equal to zero, we have

$$
-\sum_{i=1}^{n}\left(y_{i}-\sum_{j=1}^{p} \phi_{i j} X_{j}\right) \phi_{i k}+\lambda_{1} s_{k}^{(1)}+\lambda_{2} s_{k}^{(2)}=0
$$

where $\quad s_{k}^{(1)}=\partial \sum_{j=1}^{p}\left|X_{k}\right| / \partial X_{k} \quad$ and $\quad s_{k}^{(2)}=\partial \sum_{j=1}^{p} \mid X_{k}-$ $1 \mid / \partial X_{k}$. Let $r^{(k)}=\sum_{i=1}^{n}\left(y_{i}-\sum_{j \neq k} \phi_{i j} X_{j}\right)$ denote the partial residual; then using formula (A3) and after some calculations, we have

$$
X_{k}= \begin{cases}{\left[\frac{\left\langle r^{(k)}, \phi_{k}\right\rangle}{\left\langle\phi_{k}, \phi_{k}\right\rangle}+\delta_{1 k}^{*}\right]_{-},} & \frac{\left\langle r^{(k)}, \phi_{k}\right\rangle}{\left\langle\phi_{k}, \phi_{k}\right\rangle} \leqslant 0, \\ {\left[\frac{\left\langle r^{(k)}, \phi_{k}\right\rangle}{\left\langle\phi_{k}, \phi_{k}\right\rangle}-\delta_{2 k}^{*}\right]_{+},} & 0<\frac{\left\langle r^{(k)}, \phi_{k}\right\rangle}{\left\langle\phi_{k}, \phi_{k}\right\rangle} \leqslant 1+\delta_{2 k}^{*}, \\ \max \left\{1,\left[\frac{\left\langle r^{(k)}, \phi_{k}\right\rangle}{\left\langle\phi_{k}, \phi_{k}\right\rangle}-\delta_{1 k}^{*}\right]\right\}, & \frac{\left\langle r^{(k)}, \phi_{k}\right\rangle}{\left\langle\phi_{k}, \phi_{k}\right\rangle}>1+\delta_{2 k}^{*},\end{cases}
$$

where $\langle z, y\rangle$ denote the inner product of vectors $z$ and $y, \delta_{1 k}^{*}=$ $\left(\lambda_{1 k}+\lambda_{2 k}\right) /\left\langle\phi_{k}, \phi_{k}\right\rangle$, and $\delta_{2 k}^{*}=\left(\lambda_{1 k}-\lambda_{2 k}\right) /\left\langle\phi_{k}, \phi_{k}\right\rangle$. From the definition of $S_{\theta_{1}, \theta_{2}}(z)$, it is easy to see

$$
X_{k}=S_{\delta_{1 k}^{*}, \delta_{2 k}^{*}}\left(\frac{\left\langle r^{(k)}, \phi_{k}\right\rangle}{\left\langle\phi_{k}, \phi_{k}\right\rangle}\right)
$$

where the threshold function $S_{\theta_{1}, \theta_{2}}(z)$ is defined by

$$
S_{\theta_{1}, \theta_{2}}(z)= \begin{cases}\left(z+\theta_{1}\right)_{-}, & z \leqslant 0, \\ \left(z-\theta_{2}\right)_{+}, & 0<z \leqslant 1+\theta_{2}, \\ \max \left\{1, z-\theta_{1}\right\}, & z>1+\theta_{2} .\end{cases}
$$

Note that $\left\langle r^{(k)}, \phi_{k}\right\rangle=\left\langle r, \phi_{k}\right\rangle+X_{k}\left\langle\phi_{k}, \phi_{k}\right\rangle$, where $r=Y-$ $\Phi X$; we have

$$
X_{k}=S_{\delta_{1 k}^{*}, \delta_{2 k}^{*}}\left(X_{k}+\frac{\left\langle r, \phi_{k}\right\rangle}{\left\langle\phi_{k}, \phi_{k}\right\rangle}\right)
$$

Therefore, the update can be written as

$$
\hat{X}_{k}^{t+1} \leftarrow S_{\delta_{1 k}^{*}, \delta_{2 k}^{*}}\left(\hat{X}_{k}^{t}+\frac{\left\langle\hat{r}^{t}, \phi_{k}\right\rangle}{\left\langle\phi_{k}, \phi_{k}\right\rangle}\right),
$$

where $\hat{X}_{k}^{t}$ denotes the estimator of $X_{k}$ in the $t$ th step and $\hat{r}^{t}=$ $Y-\Phi \hat{X}^{t}$. The overall algorithm operates by applying this update repeatedly in a cyclical manner, updating the coordinates of $\hat{X}$ along the way. Once an initial estimator of $X$ is given, for example, by the Lasso estimation or ridge estimation, the update can be continued until convergence. The detailed algorithm based on the coordinate descent method is given in Algorithm 1, and the computational code using MATLAB and $\mathrm{R}$ software can be found on GitHub [48]. 
Algorithm 1. Algorithm for computing the signal Lasso estimation using the coordinate descent method.

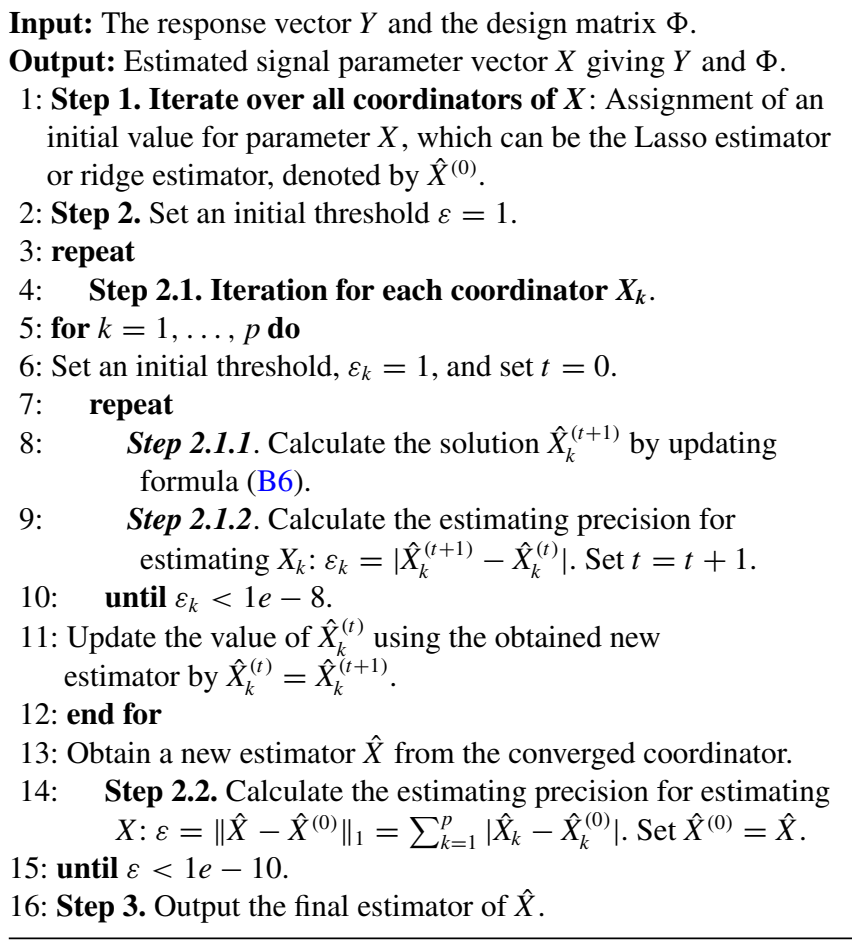

\section{APPENDIX C: THE CHOICE OF TUNING PARAMETER} by

One of the penalty functions we mentioned in (2) is given

$$
\operatorname{PF}(X, \lambda, \alpha)=\lambda_{1}\|X\|_{1}+\lambda_{2}\left\|X-\mathbf{1}_{p}\right\|_{1} .
$$

The use of $(\lambda, \alpha)$ is not much different from the use of $\left(\lambda_{1}, \lambda_{2}\right)$, but the latter is more convenient for flexible expression. Another reparameterization of the tuning parameters is that based on $\left(\delta_{1}, \delta_{2}\right)$, as shown in (A4). Obviously, there is a one-by-one transformation between $\left(\lambda_{1}, \lambda_{2}\right)$ and $\left(\delta_{1}, \delta_{2}\right)$. The reparameterization based on $\left(\delta_{1}, \delta_{2}\right)$ is more convenient, as shown in Figs. 1(b) and 1(c) in the main text. $\delta_{2}$ should not be too large since the signal Lasso will shrink $\hat{X}_{k 0}$ larger than $1+\delta_{2}$ (and less than $1+\delta_{1}$ ) toward 1 . As a result, it will shrink $\hat{X}_{k 0}<1+\delta_{2}$ (and larger than $\delta_{2}$ ) to a lower value, which means the value of $\hat{X}_{0}$ will shrink to $1-\delta_{2}$, as shown in Figs. 1(b) and 1(c). A reasonable range for $\delta_{2}$ should be in the interval $(0.1,0.4)$. The choice of $\delta_{1}$ will ensure that some larger values such as $1+\delta_{2} \leqslant \hat{X}_{k 0} \leqslant 1+\delta_{1}$ shrink to 1 and some small values such as $-\delta_{1} \leqslant \hat{X}_{k 0} \leqslant \delta_{2}$ shrink to zero depending on the situation. In a real example and simulations, we find some estimators have more negative values when there is noise in the data; larger $\delta_{1}$ is required in these special cases.

Similarly, we also can use the expression

$$
\operatorname{PF}(X, \lambda, \alpha)=\lambda\left[(1-\alpha)\|X\|_{1}+\alpha\left\|X-\mathbf{1}_{p}\right\|_{1}\right]
$$

to find the tuning parameters $\lambda$ and $\alpha$. It also involves the use of cross validation of two parameters.

The choices of tuning parameters $\left(\lambda_{1}, \lambda_{2}\right)$ or $\left(\delta_{1}, \delta_{2}\right)$ or $(\lambda, \alpha)$ can be conducted using the well-known crossvalidation technique [20]. In more detail, first, the full data set is divided into some number of groups $K>1$ (called $K$-fold). Typical choices of $K$ are 5 and 10 . If $K=n$, it corresponds to a leave-one-out test. We fix one group as the test set, and the remaining $K-1$ groups are designed the training set. We then apply the signal Lasso method based on the training set to obtain a fitted model for a range of $\left(\lambda_{1}, \lambda_{2}\right)$ and use each fitted model to predict the responses in the test set, which leads to a mean square prediction error (MSPE) for each combination of $\left(\lambda_{1}, \lambda_{2}\right)$. This procedure can be repeated $K$ times, and the average MSPE is recorded. The minimum value corresponds to a combination of $\left(\lambda_{1}, \lambda_{2}\right)$ and is selected as the best choice of tuning parameters in our method. Because two tuning parameters are involved in signal Lasso, we have to search for the best combination of $\left(\lambda_{1}, \lambda_{2}\right)$ in a two-dimensional surface for possible ranges of $\lambda_{1}$ and $\lambda_{2}$.

In our simulation studies based on the evolutionary game scheme, the observations are measured as a time series from 1 to $L$. Thus, we use the observations in time $L$ as a test set and the first $L-1$ observations as a training set. MSPE can be defined as

$$
\operatorname{MSPE}\left(\lambda_{1}, \lambda_{2}\right)=\frac{1}{N} \sum_{i=1}^{N}\left(\hat{y}_{i L}^{-L}-y_{i L}\right)^{2},
$$

where $N$ is the size of the network and $\hat{y}_{i L}^{-L}$ is a prediction of the $L$ th observation using the first $L-1$ observations in Eq. (7) of the main text. The best choice of $\left(\lambda_{1}, \lambda_{2}\right)$ will be the one that has the smallest MSPE.

\section{APPENDIX D: COMPARISON WITH THE ELASTIC NET}

The Lasso method is an effective technique for shrinkage of unimportant variables; however, as Ref. [24] pointed out, the Lasso method has some limitations. For example, in the case of the number of regression coefficients being larger than the observation number $n$, Lasso selects at most $n$ variables before it saturates. If there is a group of variables among which the pairwise correlations are very high, then Lasso tends to select only one variable from the group and does not care which one is selected. To overcome this drawback, Ref. [24] proposed a new shrink method called the elastic net, which solves the following minimization problem [20,24]:

$$
\min _{X}\left\{\frac{1}{2}\|Y-\Phi X\|_{2}^{2}+\lambda\left[\frac{(1-\alpha)}{2}\|X\|_{2}^{2}+\alpha\|X\|_{1}\right]\right\} .
$$

The penalty of the elastic net is a convex combination of the Lasso and ridge penalty. This method also has two tuning parameters; however, they both focus on shrinking $X$ towards zero. It was shown that the elastic net method performs well, especially when some variables in the model are correlated. However, the signal Lasso method proposed in this paper shrinks the parameter in two different directions ( 0 or 1$)$, having the advantage of effectively detecting signals in network reconstructions. A comparison of the simulations showing the performance of Lasso, the elastic net, and signal Lasso is given in Supplemental Material [27] in detail, which shows that signal Lasso outperforms Lasso and the elastic net in network reconstruction. 
[1] S. H. Strogatz, Exploring complex networks, Nature (London) 410, 268 (2001).

[2] R. Albert and A.-L. Barabási, Statistical mechanics of complex networks, Rev. Mod. Phys. 74, 47 (2002).

[3] D. J. Watts, Small Worlds: The Dynamics of Networks between Order and Randomness, Series: Princeton Studies in Complexity (Princeton University Press, Princeton, NJ, 2004).

[4] P. Erdös and A. Rényi, On the evolution of random graphs, in The Structure and Dynamics of Networks (Princeton University Press, Princeton, NJ, 2011), pp. 38-82.

[5] D. J. Watts and S. H. Strogatz, Collective dynamics of 'smallworld' networks, Nature (London) 393, 440 (1998).

[6] A.-L. Barabási and R. Albert, Emergence of scaling in random networks, Science 286, 509 (1999).

[7] S. Boccaletti, V. Latora, Y. Moreno, M. Chavez, and D.-U. Hwang, Complex networks: Structure and dynamics, Phys. Rep. 424, 175 (2006).

[8] P. V. Marsden, Network data and measurement, Annu. Rev. Sociol. 16, 435 (1990).

[9] C. T. Butts, Network inference, error, and informant (in) accuracy: A Bayesian approach, Soc. Networks 25, 103 (2003).

[10] T. Squartini and D. Garlaschelli, Maximum-Entropy Networks: Pattern Detection, Network Reconstruction and Graph Combinatorics (Springer, Switzerland, 2017).

[11] M. E. J. Newman, Network structure from rich but noisy data, Nat. Phys. 14, 542 (2018).

[12] T. P. Peixoto, Reconstructing Networks with Unknown and Heterogeneous Errors, Phys. Rev. X 8, 041011 (2018).

[13] T. P. Peixoto, Network Reconstruction and Community Detection from Dynamics, Phys. Rev. Lett. 123, 128301 (2019).

[14] S. G. Shandilya and M. Timme, Inferring network topology from complex dynamics, New J. Phys. 13, 013004 (2011).

[15] S. Raimondo and M. De Domenico, Measuring topological descriptors of complex networks under uncertainty, Phys. Rev. E 103, 022311 (2021).

[16] X. Han, Z. Shen, W.-X. Wang, and Z. Di, Robust Reconstruction of Complex Networks from Sparse Data, Phys. Rev. Lett. 114, 028701 (2015)

[17] W.-X. Wang, Y.-C. Lai, C. Grebogi, and J. Ye, Network Reconstruction Based on Evolutionary-Game Data via Compressive Sensing, Phys. Rev. X 1, 021021 (2011).

[18] J. Friedman, T. Hastie, and R. Tibshirani, Regularization paths for generalized linear models via coordinate descent, J. Stat. Software 33, 1 (2010).

[19] R. Tibshirani, Regression shrinkage and selection via the lasso, J. R. Stat. Soc., Ser. B 58, 267 (1996).

[20] T. Hastie, R. Tibshirani, and M. Wainwright, Statistical Learning with Sparsity: The Lasso and Generalizations (CRC Press, Boca Raton, FL, 2015), pp. 109-118.

[21] J. Fan, Y. Feng, and Y. Wu, Network exploration via the adaptive lasso and scad penalties, Ann. Appl. Stat. 3, 521 (2009).

[22] H. Zou, The adaptive lasso and its oracle properties, J. Am. Stat. Assoc. 101, 1418 (2006).

[23] F. R. Bach, Consistency of the group lasso and multiple kernel learning, J. Mach. Learn. Res. 9, 1179 (2008).

[24] H. Zou and T. Hastie, Regularization and variable selection via the elastic net, J. R. Stat. Soc., Ser. B 67, 301 (2005).

[25] D. L. Donoho, Compressed sensing, IEEE Trans. Inf. Theory 52, 1289 (2006)
[26] T. Hastie, R. Tibshirani, and J. Friedman, The Elements of Statistical Learning: Data Mining, Inference, and Prediction (Springer, New York, 2009).

[27] See Supplemental Material at http://link.aps.org/supplemental/ 10.1103/PhysRevResearch.3.043210 for the detailed model description and more simulation results for evolutionary game model, synchronization model and comparison with elastic net method.

[28] A. Szolnoki, M. Perc, and G. Szabó, Defense Mechanisms of Empathetic Players in the Spatial Ultimatum Game, Phys. Rev. Lett. 109, 078701 (2012).

[29] E. Fehr and U. Fischbacher, The nature of human altruism, Nature (London) 425, 785 (2003).

[30] G. Szabó and C. Tőke, Evolutionary prisoner's dilemma game on a square lattice, Phys. Rev. E 58, 69 (1998).

[31] G. Szabó, J. Vukov, and A. Szolnoki, Phase diagrams for an evolutionary prisoner's dilemma game on two-dimensional lattices, Phys. Rev. E 72, 047107 (2005).

[32] W. W. Zachary, An information flow model for conflict and fission in small groups, J. Anthropol. Res. 33, 452 (1977).

[33] D. Lusseau, K. Schneider, O. J. Boisseau, P. Haase, E. Slooten, and S. M. Dawson, The bottlenose dolphin community of doubtful sound features a large proportion of long-lasting associations, Behav. Ecol. Sociobiol. 54, 396 (2003).

[34] M. Girvan and M. E. J. Newman, Community structure in social and biological networks, Proc. Natl. Acad. Sci. USA 99, 7821 (2002).

[35] S. Boccaletti, G. Bianconi, R. Criado, C. I. Del Genio, J. Gómez-Gardenes, M. Romance, I. Sendina-Nadal, Z. Wang, and M. Zanin, The structure and dynamics of multilayer networks, Phys. Rep. 544, 1 (2014).

[36] M. Timme, Revealing Network Connectivity from Response Dynamics, Phys. Rev. Lett. 98, 224101 (2007).

[37] X. Wu, W. Wang, and W. X. Zheng, Inferring topologies of complex networks with hidden variables, Phys. Rev. E 86, 046106 (2012).

[38] X. Li, M. Jusup, Z. Wang, H. Li, L. Shi, B. Podobnik, H. Stanley, S. Havlin, and S. Boccaletti, Punishment diminishes the benefits of network reciprocity in social dilemma experiments, Proc. Natl. Acad. Sci. USA 115, 30 (2018).

[39] J. P. Gleeson, Binary-State Dynamics on Complex Networks: Pair Approximation and Beyond, Phys. Rev. X 3, 021004 (2013).

[40] J. Li, Z. Shen, W.-X. Wang, C. Grebogi, and Y.-C. Lai, Universal data-based method for reconstructing complex networks with binary-state dynamics, Phys. Rev. E 95, 032303 (2017).

[41] Z. Shen, W.-X. Wang, Y. Fan, Z. Di, and Y.-C. Lai, Reconstructing propagation networks with natural diversity and identifying hidden sources, Nat. Commun. 5, 4323 (2014).

[42] S. Boccaletti, C. Grebogi, Y.-C. Lai, H. Mancini, and D. Maza, The control of chaos: Theory and applications, Phys. Rep. 329, 103 (2000).

[43] T. S. Gardner, D. Di Bernardo, D. Lorenz, and J. J. Collins, Inferring genetic networks and identifying compound mode of action via expression profiling, Science 301, 102 (2003).

[44] M. Hecker, S. Lambeck, S. Toepfer, E. Van Someren, and R. Guthke, Gene regulatory network inference: Data integration in dynamic models-A review, Biosystems 96, 86 (2009). 
[45] K. Supekar, V. Menon, D. Rubin, M. Musen, and M. D. Greicius, Network analysis of intrinsic functional brain connectivity in Alzheimer's disease, PLoS Comput. Biol. 4, e1000100 (2008).

[46] L. Shi, C. Shen, Q. Shi, Z. Wang, J. Zhao, X. Li, and S. Boccaletti, Recovering network structures based on evolution- ary game dynamics via secure dimensional reduction, IEEE Trans. Network Sci. Eng. 7, 2027 (2020).

[47] A. E. Hoerl and R. W. Kennard, Ridge regression: Biased estimation for nonorthogonal problems, Technometrics 12, 55 (1970).

[48] https://github.com/shilei65/signal-lasso-code.git. 